\title{
Influencia de Simuladores y Factores Determinantes en el Bachillerato Virtual
}

\section{Influence of Simulators and Determinant Factors in the Virtual Baccalaureate}

\author{
Guadalupe Jeanette González ${ }^{1}$, Sandra Luz Canchola-Magdaleno² y Reyna Moreno-Beltran ${ }^{3}$
}

\section{(2) \\ EDICIÓN: @-CIVTAC}

Recibido: 25/junio/2021

Aceptado: 18/septiembre/2021

Publicado: 24/septiembre/2021

\section{País}

${ }^{1}$ México

${ }^{2}$ México

${ }^{3}$ México

\section{IIIII Institución}

${ }^{1}$ Universidad Autónoma de Querétaro ${ }^{2}$ Universidad Autónoma de Querétaro ${ }^{3}$ Universidad Autónoma de Querétaro

\section{$\lambda_{\Omega}$ Correo Eletrónico}

1'ggonzalez61@alumnos.uaq.mx ${ }^{2}$ andra.canchola@uaq.mx 3reyna.moreno@uaq.mx

\section{(iD) ORCID}

'https://orcid.org/0000-0002-8236-9447 ${ }^{2}$ https://orcid.org/0000-0002-7497-281X ${ }^{3}$ https://orcid.org/0000-0002-5307-0921

\section{Citar así: : APA / IEEE}

González, G., Canchola-Magdaleno, S. \& Moreno-Beltran, R. (2021). Influencia de Simuladores y Factores Determinantes en el Bachillerato Virtual. Revista Tecnológica-Educativa Docentes 2.0, 11(2), 97-103. https://doi.org/10.37843/rted.v11i2.250

G. González, S. Canchola-Magdaleno y R Moreno-Beltran, "Influencia de Simuladores y Factores Determinantes en el Bachillerato Virtual", RTED, vol. 11, n. ${ }^{\circ}$ 2, pp. 97-103, sep. 2021.

\section{Resumen}

Los simuladores son un medio tecnológico que hacen posible difundir y hacer eficiente la educación virtual con modelos educativos disruptivos. Por tal motivo, se hizo necesario investigar la pertinencia del diseño de un modelo educativo que motive el desarrollo de las habilidades STEM (ciencia, la tecnología, las ingenierías y la matemática). Este artículo tuvo el objetivo validar que el uso de simuladores permitirá promover, por medio de los programas educativos, la vocación de los egresados del nivel medio superior hacia las carreras de disciplinas STEM. El tipo de investigación presentada fue de tipo cuantitativa, de diseño no experimental - descriptiva y transversal. La metodología se basó en la aplicación de un instrumento a los egresados de bachillerato del Sistema de Universidad Virtual en la Universidad de Guadalajara. La muestra está integrada por 411 egresados, de un universo total de 1,215 egresados del Bachillerato General por Áreas Interdisciplinarias. La técnica utilizada fue el instrumento y la recolección se realizó mediante la herramienta Google forms. Esta investigación reveló factores socioculturales que influyen en el interés de los egresados en continuar sus estudios en una carrera STEM, así como la necesidad del uso de los simuladores para dar continuidad académica a las asignaturas con enfoque práctico en el contexto de la pandemia por COVID-19 o que por su naturaleza sean impartidas en línea.

Palabras clave: Simuladores, educación, STEM, bachillerato virtual.

\section{Abstract}

Simulators are a technological medium that makes virtual education possible and efficient with disruptive educational models. For this reason, it was necessary to investigate the relevance of the design of an educational model that motivates the development of STEM skills (science, technology, engineering, and mathematics). This article had the objective of validating that the use of simulators will promote, through educational programs, the vocation of graduates of the upper secondary level towards careers in STEM disciplines. The type of research presented was descriptive, and its design is non-experimental of a cross-sectional variety. The methodology was based on applying an instrument to high school graduates of the Virtual University System at the University of Guadalajara. The sample comprises 411 graduates, out of a total universe of 1,215 graduates of the General Baccalaureate by Interdisciplinary Areas. The technique used was the instrument, and the collection was carried out using the Google forms tool. This research revealed sociocultural factors that influence graduates' interest in continuing their studies in a STEM career, as well as the need to use simulators to give academic continuity to subjects with a practical approach in the context of the COVID-19 pandemic or that by their nature are taught online.

Keywords: Simulators, education, STEM, virtual high school. 


\section{Introducción}

Los simuladores son un medio tecnológico que hacen posible difundir $\mathrm{y}$ hacer eficiente la educación virtual con modelos educativos disruptivos. La educación STEM (acrónimo en inglés de los términos Science, Technology, Engineering y Mathematics) fue acuñada por la National Science Fundation (NSF) en los años noventa. En el ámbito académico, el aprendizaje STEM tomó impulso entre 2005 y 2010; después de su éxito en países de primer mundo, varios autores exhortaron a una mejora de la enseñanza en estas disciplinas para impulsar el crecimiento de países en desarrollo (Giraldo, Molina y Córdoba, 2018). El estudio de Yildirim (2016) mostró que incluir aprendizajes STEM en la trayectoria académica en niveles básicos e intermedios fomenta la elección de las profesiones relacionadas con la ciencia y la tecnología.

Al tomar el placer y la creatividad como detonantes fundamentales del aprendizaje, Zimmerman (2016) mostró en su investigación una tabla comparativa con diferentes insumos para impulsar en los estudiantes las habilidades STEM, que incluye el uso de laboratorios y simuladores como las herramientas educativas más eficientes utilizadas en países desarrollados. Los simuladores son actualmente una tendencia formativa a nivel internacional que opera como parte de los modelos disruptivos respecto a la escuela convencional, los cuales favorecen la integración de competencias digitales y de innovación en cualquier campo del conocimiento (Toulmin \& Groome, 2017).

Fundamentado en lo anterior y debido a la importancia de fortalecer el aprendizaje de las matemáticas, la ciencia y la tecnología en los planes de estudios del nivel medio superior del Sistema de Universidad Virtual, se hizo necesario investigar la pertinencia del diseño de un modelo educativo que motive el desarrollo de las habilidades STEM.
Con la finalidad de que el uso de simuladores cumpla con el objetivo de generar el interés por las disciplinas STEM en los alumnos y de complementar adecuadamente el diseño instruccional de las asignaturas que los utilicen, se exploró la influencia en la elección de carreras STEM que tienen diversos factores socioculturales mencionados en la literatura tales como: referentes familiares, grado académico de los padres (Avendaño, Magaña \& Flores, 2020), clases extracurriculares (Saldaña, 2021), tecnología y género (Pineda, 2021), estereotipos de la infancia (Giraldo, Molina \& Córdoba, 2018) dificultad con las matemáticas (Cabrero, 2015), entre otros.

Una vez comprobada su pertinencia los laboratorios virtuales de simuladores, estos se desplegaron como un espacio que se caracteriza por la promoción de la investigación interdisciplinaria y además facilitaron la aplicación práctica de las disciplinas abordadas (Zawieska \& Duffy, 2015). Este artículo tuvo el objetivo validar que el uso de simuladores permitieron promover, por medio de los programas educativos, la vocación de los egresados del nivel medio superior hacia las carreras de disciplinas como la ciencia, la tecnología, las ingenierías y la matemática (conocidas por sus siglas en inglés como STEM).

\section{Metodología}

El positivismo es un paradigma que guían la investigación cuantitativa, los cuales tienen como objeto explicar el fenómeno estudiado, para en una última instancia, predecirlo y controlarlo (Guba \& Lincoln, 2002). Hernandez et al. (2003), clasifican al diseño de investigación no experimental como la investigación que se realiza sin manipular deliberadamente variables y en los que solo se observan los fenómenos en su ambiente natural para después analizarlos. Asimismo, para Hernandez et al. (2003), el diseño no experimental se divide tomando en cuenta el tiempo durante los datos, estos son transversal donde se recolectan datos en un 
Recursos didácticos según de Guy Brousseau para fortalecer la división de números naturales en estudiantes del grado quinto de la Institución Educativa Padua a través de una secuencia de actividades didáctica.

solo momento, en un tiempo único, además su propósito es describir variables y su incidencia de interacción en un momento dado.

El diseño descriptivo en una investigación busca caracterizar, exponer, describir, presentar o identificar aspectos propios de una determinada variable (Arguedas, 2009). Para fines de este estudio se realizó un diseño no experimentaltransversal, debido a que se recolectaron datos en tiempo determinado sin intervenir ene 1 ambiente en que se desarrollaron.

Los datos se recolectaron desde un instrumento en línea por medio de la herramienta Google forms. El cuestionario se integró con preguntas que ayudaron a identificar si existe algún tema, asignatura o clase extracurricular que detonara el interés de los egresados por alguna carrera STEM, si el egresado conoce qué es la educación STEM y si cuenta con un referente en su familia que sea profesional en estas disciplinas, así como el grado académico de los padres y sus profesiones, en caso de tenerlas. Se cuestionaron también las materias que les representaron mayor dificultad y el actual dominio matemático de los egresados. Así mismo, en este instrumento se recolecta información sobre la carrera que cursan actualmente, en caso de que hayan decidido continuar con sus estudios.

La muestra está integrada con total de 411 egresados, de un universo total de 1,215 egresados del Bachillerato General por Áreas Interdisciplinarias, del Sistema de Universidad Virtual de la Universidad de Guadalajara, el cual es un órgano desconcentrado de la Universidad de Guadalajara responsable de administrar y desarrollar programas académicos de nivel medio superior y superior, en modalidades no escolarizadas, apoyadas en las tecnologías de la información y de la comunicación. Se obtuvo un nivel de confianza del $95 \%$ en la investigación, con un margen de error del $4 \%$. Para el procesamiento de los datos se aplicó un análisis multivariable de varianza y pruebas T con el software SPSS 25.

\section{Modelo educativo del Sistema de Universidad Virtual}

Se identifico que la edad promedio de los egresados en modalidad virtual es de 42 años, esto obedece a que de acuerdo con el modelo educativo está dirigido a personas que no tienen la educación como actividad principal y optaron por la modalidad virtual para continuar con su formación académica (Moreno, Chan, Flores, Pérez, Ortiz, Hernández \& Coronado, 2010), con un 63\% de egresados de sexo masculino y un $37 \%$ de sexo femenino, pues la mayoría de los egresados hombres tenían un trabajo como actividad principal. En el caso de las mujeres que egresan del bachillerato se puede observar un menor porcentaje, pues dan prioridad a las actividades domésticas o de cuidados una vez terminada su jornada laboral de acuerdo con los roles impuestos desde la infancia según los autores Giraldo, Molina \& Córdoba (2018).

Para más de la mitad de los alumnos encuestados la asignatura de matemáticas fue la que representó mayor dificultad, seguida de química y tecnologías de la información, como se muestra en la Figura 1. Un 98\% de los egresados considera que el aprendizaje en estas asignaturas se hubiera facilitado con la implementación de laboratorios virtuales de simuladores para prácticas donde se brindará la posibilidad aplicar el conocimiento adquirido de forma teórica.

\section{Figura 1 \\ Asignaturas con mayor dificultad en el} bachillerato modalidad virtual

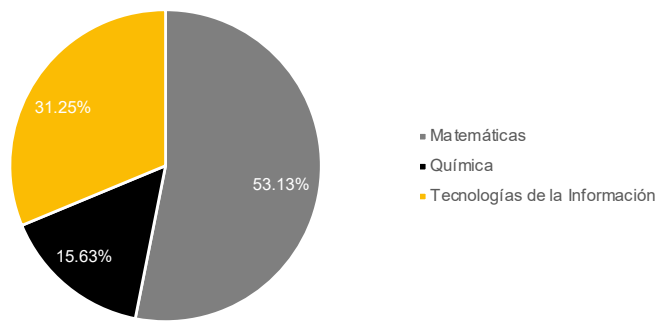

Nota. Asignaturas que representaron mayor dificultad para los egresados de bachillerato del Sistema de Universidad Virtual, elaboración propia (2021)

\section{Resultados}


Recursos didácticos según de Guy Brousseau para fortalecer la división de números naturales en estudiantes del grado quinto de la Institución Educativa Padua a través de una secuencia de actividades didáctica.

A un $90 \%$ de los egresados se les dificultan las matemáticas actualmente, ya que las siguen considerando muy difíciles lo que demuestra lo afirmado por Yildirim (2016) en su tesis doctoral donde considera que los métodos de enseñanza de las matemáticas siguen siendo obsoletos en las diversas modalidades educativas. El 65\% de los egresados que decidieron continuar sus estudios prefirieron continuar de forma presencial, ya que expusieron como causas principales: la dificultad organizar los tiempos que dedicaban al estudio al no ser su actividad principal y el modelo de asesor del docente planteado por el modelo educativo del Sistema de Universidad Virtual (Moreno, Chan, Flores, Pérez, Ortiz, Hernández \& Coronado, 2010), pues los egresados consideraron en un $75 \%$ que el poco acompañamiento docente fue una limitante para que se tuviera preferencia por la modalidad virtual para dar continuidad a los estudios, datos expuestos en la Figura 2 y Tabla 1.

\section{Figura 2}

Modalidad de preferencia para continuar con sus estudios

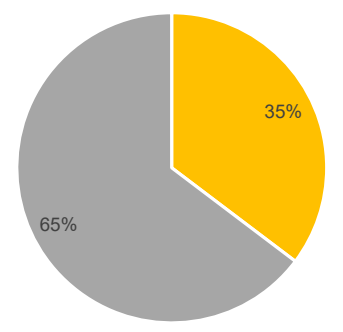

- EN LINEA -PRESENCIAL

Nota. Preferencia de modalidad para continuar los estudios de licenciatura, elaboración propia (2021).

\section{Tabla 1}

Causas por las que un egresado no elige continuar sus estudios en la modalidad virtual

\begin{tabular}{ll}
\hline CAUSA $\boldsymbol{P O R C E N T A J E}$ \\
\hline Tiempo de dedicación & $40 \%$ \\
\hline $\begin{array}{l}\text { Poco acompañamiento } \\
\text { docente }\end{array}$ & $38 \%$ \\
\hline Costo & $8.2 \%$ \\
\hline Poca oferta educativa & $3.5 \%$ \\
\hline $\begin{array}{l}\text { Nota. Causas por las que los egresados de bachillerato } \\
\text { del Sistema de Universidad Virtual de la Universidad } \\
\text { de Guadalajara deciden no continuar sus estudios en } \\
\text { modalidad virtual, elaboración propia (2021). }\end{array}$
\end{tabular}

Lo anterior coincide con lo expuesto por Zornoza (2016) quien afirma que en estos tiempos el uso de la tecnología permite al estudiante la interacción con otros actores como docentes y compañeros, análisis y modelación de la realidad o una parte de ésta, con la posibilidad de retroalimentación inmediata y de ir más haya un aprendizaje basado en procesos y procedimientos; esto representa un avance metodológico y metacognitivo ante las descontextualizadas actividades educativas.

\section{Elección de carreras STEM}

De los alumnos egresados analizados, la mitad decidieron no estudiar una licenciatura (Tabla 2) y solo 123 cursan una carrera STEM, lo que representa un $31.6 \%$ de los encuestados, como lo muestra la Tabla 3 y la Figura 3.

\section{Tabla 2}

Carreras cursadas por egresados de bachillerato

\begin{tabular}{|l|c|}
\hline \multicolumn{1}{|c|}{ CARRERA } & EGRESADOS \\
\hline NO ESTUDIA & 207 \\
\hline TECNOLOGÍAS & 76 \\
\hline LIC. MATEMÁTICAS & 8 \\
\hline CIENCIAS & 39 \\
\hline OTRA CARRERA & 81 \\
\hline
\end{tabular}

Nota. Tabla de carreras cursadas actualmente por los egresados de bachillerato del Sistema de Universidad Virtual de la Universidad de Guadalajara, elaboración propia (2021).

\section{Figura 3}

\section{Carreras cursadas por egresados}
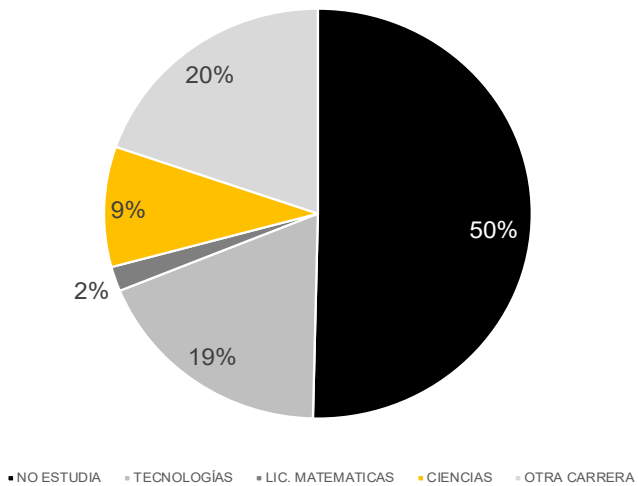

Nota. Gráfica carreras cursadas actualmente, elaboración propia (2021). 
Recursos didácticos según de Guy Brousseau para fortalecer la división de números naturales en estudiantes del grado quinto de la Institución Educativa Padua a través de una secuencia de actividades didáctica.

\section{曲}

Los egresados que cursan una carrera STEM coinciden en $84 \%$ que el detonante de su interés por estas disciplinas fue la materia Tecnologías de la Información, seguida de Matemática y Ciencia en un 9\% y de Física y razonamiento científico en un $7 \%$. Tomando en cuenta que estas materias contienen un diseño instruccional basado en casos y tienen apoyo de recursos tecnológicos, se puede afirmar lo expuesto por Pineda-Castillo (2021) que la aplicación práctica de los conocimientos teóricos con apoyo tecnológico favorece el aprendizaje y comprensión de asignaturas STEM.

Es importante mencionar que un $98 \%$ de los egresados que están cursando una licenciatura no cuenta con laboratorios virtuales para dar continuidad a sus asignaturas prácticas sin importar la modalidad elegida aún en tiempos de pandemia, lo que los egresados suponen ha dificultado continuar con el aprendizaje.

\section{Influencia de la familia, género y otros factores en la elección de carreras STEM}

De los egresados que cursan una licenciatura STEM, solo 36 son mujeres de las cuales solo el $2 \%$ tiene hijos en edad escolar, un total del $29.26 \%$ de los egresados que han continuado sus estudios y un $8.7 \%$ del total de los egresados como se muestra en la Figura 4.

\section{Figura 4}

Género de los egresados en carreras STEM

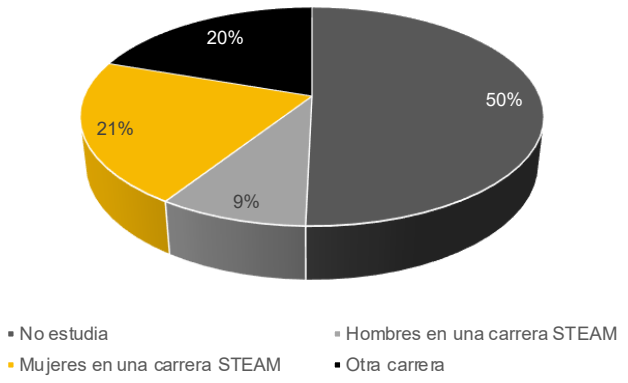

Nota. Gráfica de género de egresados de bachillerato del Sistema de Universidad Virtual de la Universidad de Guadalajara, elaboración propia (2021).

Del $21 \%$ de los estudiantes que no continuaron sus estudios un $73 \%$ es mujer y

un $98.3 \%$ dedica la mayor parte de su tiempo al trabajo doméstico y al de cuidados de adultos mayores o menores en edad escolar, demostrando que los estereotipos de la infancia influyen directamente en la continuidad de los estudios como afirman los investigadores Giraldo, Molina y Córdoba (2018).

A pesar de que la sociedad se encuentra en una transición cultural, intelectual, social y tecnológica que tiene un gran impacto en los roles y composiciones familiares; aunque en los últimos tiempos ha aumentado la participación de la mujer en posiciones de poder en ámbitos donde anteriormente no figuraba, su incursión en la ciencia sigue siendo muy bajo de acuerdo con la Unesco (2019). Lo anterior debido a que las mujeres siguen evitando elegir carreras que demanden largas jornadas laborales que limiten el ejercicio de su maternidad y a los estereotipos familiares.

De los egresados que cursan o cursaron una carrera STEM, el 98\% cuentan con un referente en su familia que es profesionista en estas disciplinas, y en un $60 \%$ es el padre o un hermano. Solo un solo un $30 \%$ de los egresados conocía el término STEM. Con los datos mencionados se demuestra que el concomiendo STEM que posee el núcleo familiar es una influencia positiva para la elección de una carrera en estas disciplinas, coincidente con la investigación de Avendaño, Magaña \& Flores (2020).

El $86 \%$ de los alumnos que eligieron continuar sus estudios en una carrera de esta disciplina, tienen una madre cuyo nivel académico mínimo es de bachillerato y en un $94 \%$ ella aún vive con la o el estudiante o lo hacía recientemente. Solo el $14 \%$ de los egresados cursando una licenciatura en estas disciplinas tomó clases extracurriculares.

Se comprueba con este estudio que el nivel educativo de los padres tiene una influencia significativa en $\mathrm{su}$ trayectoria escolar. En este estudio siempre la influencia de la madre en la elección de una carrera fue mayor a cualquier otro familiar, lo que se contrapone con el estudio realizado por Avendaño, Magaña \& Flores (2020) quienes afirman que el hecho de vivir con la madre es un único factor de influencia en la elección de 
Recursos didácticos según de Guy Brousseau para fortalecer la división de números naturales en estudiantes del grado quinto de la Institución Educativa Padua a través de una secuencia de actividades didáctica.

carrera del estudiante. Solo el $14 \%$ de los egresados cursando una licenciatura en estas disciplinas tomó clases extracurriculares lo que no es coherente con la investigación de Saldaña (2021) quien afirma que tienen fuerte influencia en la elección de carrera de un estudiante de bachillerato.

\section{Conclusiones}

Esta investigación demostró la total pertinencia de la implementación de los laboratorios virtuales con simuladores, por lo menos desde el punto de vista de los egresados. En trabajos futuros, se podría investigar su pertinencia específica para las asignaturas de matemáticas, química $\mathrm{y}$ tecnologías, debido a la dificultad que estas presentaron para los egresados. Se sugiere revisar a detalle el diseño instruccional actual de la asignatura de matemáticas, ya que los egresados coincidieron en su alta dificultad.

La pandemia por COVID-19 dejo al descubierto la necesidad de una modalidad hibrida permanente en la educación; habiendo comprobado que los egresados de bachillerato consideran que el uso de simuladores hubiera facilitado su aprendizaje en materias STEM solo revisadas de forma teórica que representaron mayor dificultad a lo largo de su bachillerato, se puede predecir que el uso de esta tecnología tenderá a normalizarse en todos los niveles de la educación. A pesar de lo anterior, actualmente los simuladores continúan sin ser utilizados comúnmente en las diferentes modalidades de la educación, se recomienda que en trabajos futuros se profundice en las razones por las que sucede esta situación.

Será pertinente analizar en investigaciones posteriores si utilizar simuladores en las asignaturas que representaron mayor dificultad de aprendizaje puede detonar una mayor preferencia por continuar sus estudios de licenciatura en línea.

Se demostró que el contenido actual del programa de Bachillerato del Sistema de Universidad Virtual genera poco interés en disciplinas STEM y que el generado fue particularmente en el área de Tecnologías de la información a pesar de que se consideró una de las asignaturas con mayor dificultad, fue relevante el uso de tecnología educativa como apoyo en el diseño instruccional. Así mismo el interés motivado en las mujeres para cursar alguna licenciatura STEM o incluso para continuar sus estudios es muy bajo por lo que se propone continuar con investigaciones para incentivar la participación de la mujer y concientizar a los empleadores de los egresados de carreras en estas disciplinas en flexibilizar las jornadas laborales y garantizar la equidad de género en sus centros de trabajo.

Fue necesario para los egresados contar con un referente familiar que estuviera ejerciendo alguna profesión STEM para que pudieran elegir dar continuidad a sus estudios en una disciplina similar; esté referente la mayoría de las veces fue el padre o el hermano. El nivel académico de la madre y el que cohabitara con el estudiante fue particularmente relevante y representó una influencia para la continuidad de los estudios en estas disciplinas. No fue relevante cursar clases extracurriculares para motivar el interés en continuar una educación STEM.

\section{Referencias}

Arguedas, O. (2009). La pregunta de investigación. Acta Médica Costarricense 51 (2), 89-90.

Avendaño-Rodríguez, K. C., Magaña-Medina, D. E. \& Flores-Crespo, P. (2020). Influencia familiar en la elección de carreras STEM (Ciencia, tecnología, ingeniería y matemáticas) en estudiantes de bachillerato. Revista de Investigación Educativa, 38(2) $515-531$.

https://doi.org/10.6018/rie.366311

Hernández-Sampierí, R.; Fernández-Collado, C. \& BaptistaLucio, P. (2003). Metodología de la investigación, 3. ed. McGraw-Hill.

Giraldo, F., Molina, J \& Córdoba, F. (2018). (Compls.). Experiencias de investigación en escenarios escolares. Elementos para una transformación educativa y social. Instituto Tecnológico Metropolitano.

Guba, E., \& Lincoln, Y. (2002). Paradigmas en competencia en la investigación cualitativa. En Derman, C. \& Haro, J. Por los rincones. Antología de métodos cualitativos en la investigación social. El Colegio Sonora.

Pineda-Castillo, K. A. (2021). Uso de Tecnología como Recurso Preponderante en el Aprendizaje a Distancia en tiempos de Confinamiento Social. Revista Tecnológica-Educativa Docentes 2.0, 11(1), 89-98. https://doi.org/10.37843/rted.v11i1.197 
Recursos didácticos según de Guy Brousseau para fortalecer la división de números naturales en estudiantes del grado quinto de la Institución Educativa Padua a través de una secuencia de actividades didáctica.

Moreno-Castañeda, M., Chan-Núñez, M., Flores-Briseño, M., Pérez-Alcalá, M. S., Ortiz-Ortiz, M. G., Hernández-Figueroa, V. G., ... \& CoronadoRamírez, G. (2010). Modelo educativo del Sistema de Universidad Virtual. México: Sistema de Universidad Virtual. Universidad de Guadalajara.

Martínez-Sanz, R., Islas-Carmona, O., CamposDomínguez, E. \& Redondo-García, M. (2016). El profesor universitario de Comunicación: acceso, consumo y cultura mediática. Un estudio comparativo entre España y Méxic. Revista Latina de Comunicación Social, 71, 349372.DOI: 10.4185/RLCS-2016-1099

Saldaña-Acosta, J. M. (2021). Desarrollo de Actitudes de Colaboración, Cooperación e Innovadoras en el Aula Basado en Proyectos. Revista TecnológicaEducativa Docentes 2.0,11(1), 130-139. https://doi.org/10.37843/rted.v11i1.201

Toulmin, C. N. \& Groome, M. (2017). Building a science, technology, engineering, and math agenda. National Governors Association.

Organización de las Naciones Unidas para la Educación, la Ciencia y la Cultura (Unesco). (2019). Women in Science.

http://uis.unesco.org/sites/default/files/documents/f s55-women-in-science-2019-en.pdf

Zimmerman, A. (2016). Developing confidence in steam: exploring the challenges that novice elementary teachers face. The STEAM Journal. 2 (2), 1-11. https://scholarship.claremont.edu/cgi/viewcontent.c gi?referer=https://www.google.com/\&h ttpsredir $=1 \&$ article $=1122 \&$ context $=$ steam

Zornoza-Martínez, E. (2016). Aprendizaje con simuladores. Aplicación a las Redes de Comunicaciones. Revista de Nuevas Tecnologías y Sociedad, (42). $\mathrm{http}: / /$ dialnet.unirioja.es/servlet/articulo? codigo $=19$ 60039 